\title{
Malaysian Medical Device Regulation for Artificial Intelligence in Healthcare: Have all the pieces fallen into position?
}

\author{
Nurus Sakinatul Fikriah Mohd Shith Putera', Sarah Munirah Abdullah' \\ Noraiza Abd Rahman ${ }^{1}$, RafizahAbu Hassan ${ }^{1}$, Hartini Saripan ${ }^{1}$, Imam Haryanto ${ }^{2}$ \\ ${ }^{1}$ Faculty of Law, Universiti Teknologi MARA, Shah Alam, Malaysia \\ ${ }^{2}$ Faculty of Law, Universitas Pembangunan Nasional Veteran, Jakarta, Indonesia \\ nurussakinatul@uitm.edu.my,sarahmunirah@uitm.edu.my, \\ noraizarahman@uitm.edu.my, fiza@uitm.edu,my, hartinisaripan@uitm.edu.my, imam.haryanto@upnvj.ac.id:
} Tel: +60195778006:

\begin{abstract}
Artificial Intelligence $(\mathrm{Al})$ ability of self-learning and adaptation has challenged the medical device regulation in overseeing the safety and effectiveness of medical devices. Thus, this research aims to evaluate the adequacy of the pre-market requirements under the Medical Device Act 2012 in governing Al modification. Employing the doctrinal research methodology, systematic means of legal reasoning pertinent to Al for healthcare applications are produced. An effective medical device regulation is pivotal to foster trustworthiness in the governance and adoption of Al. However, the research findings indicate the deficiency of the current conformity assessment for medical devices in addressing Al modifications.
\end{abstract}

Keywords: Artificial Intelligence and Law, Artificial Intelligence and Medical Device Regulation, Malaysian Medical Device Regulation

eISSN: 2398-4287@ 2021. The Authors. Published for AMER ABRA cE-Bs by e-International Publishing House, Ltd., UK. This is an open access article under the CC BYNC-ND license (http://creativecommons.org/licenses/by-nc-nd/4.0). Peer-review under responsibility of AMER (Association of Malaysian Environment-Behaviour Researchers), ABRA (Association of Behavioural Researchers on Asians/Africans/Arabians) and CE-Bs (Centre for Environment-Behaviour Studies), Faculty of Architecture, Planning \& Surveying, Universiti Teknologi MARA, Malaysia. DOI: https://doi.org/10.21834/ebpj.v6i16.2635

\subsection{Introduction}

Artificial Intelligence (Al) once seen as science fiction. Nevertheless, Al is now taking a leading role in revolutionising many industries, including the healthcare industry, capitalising on the vast swathes of data generated during healthcare delivery. Various medical specialities, ranging from radiology and oncology to ophthalmology and general medical decision-making, have integrated Al within general practices as this technology exponentially improves medical outcomes. Many challenges faced by health practitioners in providing treatment coverage to patients resolved by adopting Al-driven digital tools. Amongst these challenges are difficulty in handling explosions of digital data and the discovery of novel diseases. Able to replicate human cognitive functions, Al may perform as well, if not better than the healthcare practitioners, augmenting the healthcare practitioners' efforts in, among others, making more accurate diagnoses, identifying at-risk populations, managing and better understanding patients' responses to medicines and treatment protocols. As healthcare practitioners are supplied with a massive amount of data on patients within the healthcare setting, Al can trace the most relevant clinical information in assisting the practitioners in making clinical decisions (Jiang F, Jiang Y, Zhi H, 2017). For instance, in emergency medical services, the Al algorithm has been proven to surpass the conventional triage tools and early warning scores in determining patients' need for critical care (Meskó, 2020). A crucial aspect of healthcare delivery, which is the diagnosis and treatment

eISSN: 2398-42870 2021. The Authors. Published for AMER ABRA cE-Bs by e-International Publishing House, Ltd., UK. This is an open access article under the CC BYNC-ND license (http://creativecommons.org/licenses/by-nc-nd/4.0). Peer-review under responsibility of AMER (Association of Malaysian Environment-Behaviour Researchers), ABRA (Association of Behavioural Researchers on Asians/Africans/Arabians) and CE-Bs (Centre for Environment-Behaviour Studies), Faculty of Architecture, Planning \& Surveying, Universiti Teknologi MARA, Malaysia.

DOI: https://doi.org/10.21834/ebpj.v6i16.2635 
of diseases, has long been a focus of Al since the 1970s (Davenport \& Kalakota, 2019). More recently, as it improves, IBM Watson, among the pioneer Al systems, has been used in designing treatment plans for patients. With the advent of this Al-integrated systems, the process of determining patient treatment pathways which traditionally takes days to complete has now been able to be sped up to just a few minutes (Meskó, 2020).

This would be crucial, for example, in tackling diseases which progress rapidly and can cause complications, such as leukaemia. With the aid of IBM Watson, the oncologists at Institute of Medical Science, the University of Tokyo in Japan discovered a rare type of secondary leukaemia in a patient which eventually led the team to the patient's life-saving treatment (Otake, 2016). Meanwhile, in cancer research conducted by the University of North Carolina School Of Medicine, 99 per cent of IBM Watson treatment recommendations were consistent with those suggested by the oncologists. In 30 per cent of these cases, IBM Watson has outperformed the oncologists in discovering treatment options not identified by the oncologists due to adaptation based on cancer research papers and recent clinical trial not accessible to the oncologists (Lohr, 2016). On the other hand, within radiology, Al also performs better than the radiologists in detecting malignant tumours (Savage, 2020). In 2016, the Moorfields Eye Hospital NHS Foundation Trust in England announced a joint venture with Google Deepmind Health in ophthalmology. The collaboration has enabled the improvement of eye treatment through analysis of retina scans by Deepmind algorithms. As a result, within just thirty seconds, diseases such as diabetic retinopathy, glaucoma and age-related macular degeneration can be detected. Another area worth paying attention to concerning Al contribution to the healthcare industry is the drug creation aspect. The process which often takes months or even years to complete and involve exorbitant costs of up to billions of dollars may be concluded more speedily and cost-effectively with the help of Al. A company, Atomwise, while using its Al technology managed to discover two new drugs meant to reduce the infectivity of Ebola in just two days (Meskó, 2020). Another example of Al application in healthcare settings points to health monitoring and medication management, particularly relevant post-diagnosis and treatment pathways design. Sense.ly's Molly, a virtual nurse, carries out these tasks by providing personalised follow-up care to monitor and manage chronic diseases in between doctor visits. AiCure, on the other hand, a smartphone app, uses the same approach in employing Al to keep track of patient adherence to prescriptions (Meskó, 2020). Al contribution to the healthcare industry is revolutionary, but not without a price. More mundanely, Al has challenged the way scientific understanding and validation of medical technologies operate, precisely, within the framework of medical device regulation. Instances of Al producing medical recommendations beyond what humans can comprehend, much less to employ, merit a revisit of the aptness of the regulatory pathways for Al. This research, thus, aims to advance the discussion as to the feasibility of the prevailing medical devices pre-market evaluation under the Malaysian Medical Device Act 2012 for Al. The objective of this research is to improve the Malaysian medical device regulation by comprehending the adaptive nature of Al systems which deviate from the conventional medical devices. This research also seeks to learn some lessons from the United States approaches in regulating Al for healthcare purposes, precisely on the application of the Total Product Life Cycle approach.

\subsection{Literature Review}

\subsection{The Medical Device Regulation}

Throughout the product lifecycle of a medical device, regulatory affairs practitioners serve a vital role, guiding pre-market policy, drafting regulatory submissions, and ensuring post-market compliance (Shiffman, 2018). Al is labelled with its ability to self-learn from timely data it acquires from real-world applications, placing it amongst software as a medical device (SaMD), an evolving field of research and development in the medical device regulation (Benjamens et al., 2020). Taken at face value, the regulatory controls aim to safeguard the health and safety of patients, consumers and others by ensuring that medical device manufacturers comply with the prescribed procedures during design, manufacture and marketing. The safety and performance of medical devices depend on two critical elements adopted by the medical device regulation which are the pre-market review and the post market surveillance (World Health Organisation, 2015). While the former focuses on product control, the latter guarantees that medical devices in use remain to be safe and effective. The two critical elements can be better perceived through the Life Span diagram below:

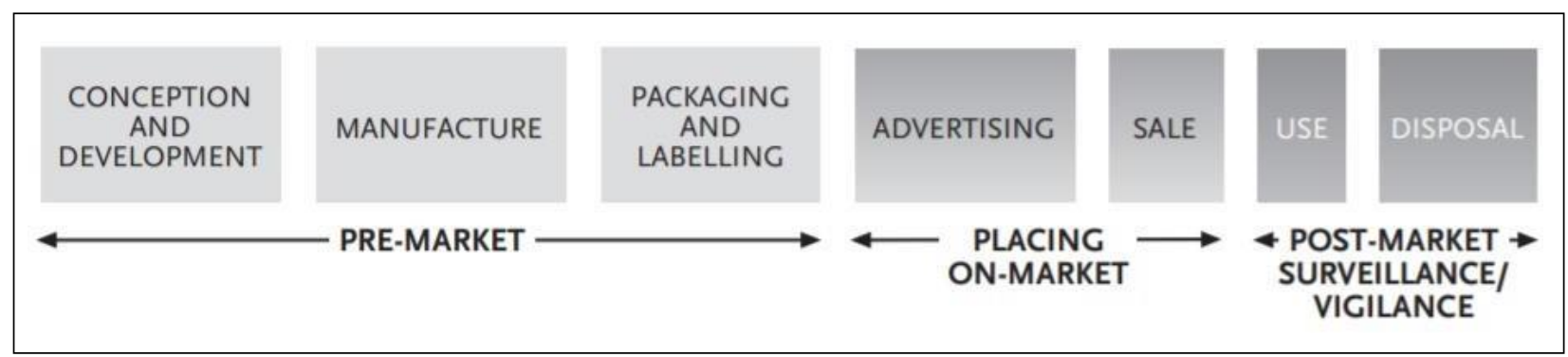

Fig. 1: Common Elements of Medical Device Regulation (World Health Organisation, 2015)

The elements or components for medical device regulation are universally adopted by countries in the world including Malaysia and the United States. Arguably, one of the issues that has raised concerns over the use Al-fuelled medical device is that the level of controls would depend on the defined risks associated with devices. Plus, an essential aspect of any regulatory control framework is identifying an effective way of producing a sustainable set of regulations (Medical Device Authority, 2014). The classification of medical devices in 138 
Malaysia is mainly based on the risk associated with the human body vulnerability, the technological design and development of medical devices, which take into consideration the intended use, the transient duration of use and also the part of the human body (Medical Device Authority, 2014). Under these rules, the actual classification of each device relies on the claims made by the manufacturer and on its intended use. While interpreting these rules, it must be stressed that the actual classification of a specific device must be considered individually, considering its nature and intended usage. Despite providing the rules of classification for general medical devices, insignificant attention is given to the classification of SaMD by the Malaysian Medical Device Authority (MDA), resulting with a reliance towards the international risk categorisation framework discussed below.

\subsection{Risk Categorisation and Pre-Market Requirements}

The International Medical Device Regulators Forum (IMDRF) Software as a Medical Device Working Group (WG) has released an External Link Disclaimer for Software as a Medical Device, a potential risk categorisation framework. Ideally, the recommendations provided in this document will help the manufacturers and regulators to define risk categories of Software as a Medical Device more specifically based on how the performance of a Software as a Medical Device is used in various healthcare circumstances or conditions for healthcare decisions (US Food and Drugs Administration, 2017). The IMDRF also recommends a set of standards to be implemented in the SaMD risk categorisation sense (IMDRF Framework for SaMD Risk Categorisation, 2020) which can be extended to AI SaMD. It is important to emphasise that the Software as a Medical Device risk categorisation framework External Link Disclaimer has four categories (I, II, III, and IV). These categories are based on patient or public health effect levels where reliable information given by the Software as a Medical Device to treat or diagnose, drive or advise clinical management is essential and pertinent to prevent death, longterm disability or severe other degradation in health, reduce public health (IMDRF SaMD Working Group, 2017). The Level IV category is Software as a Medical Device with the highest impact on the patient or public health. Level I is the lowest. As prescribed in the Medical Device Act 2012, the pre-market requirements are the Malaysian Medical Device Authority (MDA) process of scientific and regulatory review to evaluate the safety and effectiveness of the classes of medical devices before market placement (Medical Device Authority, 2014). Section 5 of the 2012 Act Act outlines the manufacturer's responsibility to ensure that a medical device conforms to the defined Essential Principle of Safety and Performance (EPSP), that a medical device's production is certified with the Good Manufacturing Practice (GMP) or with any written directives issued by the MDA, and is packaged, labelled and marked in an approved manner. Following the verification by the Conformity Assessment Body $(C A B)$ certifying that the conformity assessment is conducted in adherence to the requirements under the Act, the MDA in return will register the medical device (Section 7(1)). Likewise, in the United States, to ensure circulation and access to safe medical devices within the society, the Food and Drugs Administration (FDA) adopts a risk-based three-class scheme in regulating medical devices. The three regulatory classes embedded in the 1976 Medical Device Amendment are: class I - low risk; class II - moderate risk; and class III - high risk. The devices will then be controlled differently based on factors such as their "intended purpose" and "indication of purpose". These factors would determine the type of marketing application prior to registering the robots with the FDA (Jarow \& Baxley, 2015). The "life cycle concept" adopted by FDA is imperative in guaranteeing that the medical devices meet the standard of approval required for marketing purposes. This concept comprises all stages of medical devices production and use from pre-market requirement, placing of the device in the market until post-market control. Manufacturers of non-exempt devices are required to submit to the FDA either a Pre-Market Approval (PMA) application where FDA would either outright approve the device, or a Pre-Market Notification where the device would be cleared if it is significantly similar to the predicate devices. In arriving at these decisions, the FDA is guided by the risk demonstrated by the device through information and scientific evidence submitted by the manufacturer (Kramer et al., 2012). Prior to each submission, the manufacturers are given opportunities to engage in discussions with the FDA with regard to the device to support the marketing application. From the literature, an area that has raised concern is that hazard identification and safety metrics should be context-specific in the sense that the technical understanding of a particular technology is prioritized. Undoubtedly, the pre-market requirements for medical devices applicable to Al facilitates in providing certain and a predictable regulatory environment to manufacturers in positioning healthcare devices into the market. However, subjecting $\mathrm{Al}$ to the medical device regulation results with the generalisation of the governing rules as early as defining Al as a medical device. If the medical device regulation appears to be inefficient in assuring safe and effective medical devices, it is quite impractical to evaluate whether 'unfitness' implies to be unfit for the medical devices largely or Al specifically. For instance, the rising number of lawsuits filed against the Intuitive Inc. concerning the defective Da Vinci Surgical System, a predecessor technology to Al, notwithstanding adhering to the regulatory requirement required of a medical device outlined by the FDA, questions arise as to whether the medical device regulation pertaining class II medical device is unworkable or whether the medical device regulation is insignificant to medical robots altogether. This question will certainly bleed into the regulation of Al within the medical device framework.

\subsection{Methodology}

This research considers its activities to that of a legal-interdisciplinary interest, an approach pivotal to the development of legal arguments by obtaining input from other disciplines of knowledge, particularly for this research, the field of Artificial Intelligence and medical device framework (Naudé Fourie, 2015). In the context of this research, the doctrinal analysis is employed to synthesise various statutory provisions, regulatory principles, interpretive guidelines and framework (Hutchinson \& Duncan, 2014) related to Artificial Intelligence in the frame of medical device framework. The principal purpose of this method is also explored in elucidating normative critics for the formulation of proposals for the future endeavours. As much as this research is concerned, the sources for the doctrinal 
analysis approach are captured in written sources such as statutory legislation, case law, regulation guideline documents, journal articles and reports retrieved from a library-based search. The library-based search was aided by the UiTM Online Public Access Catalog (OPAC) system to identify primary data such as the Medical Device Act 2012, the Malaysian Contracts Act 1950, the Consumer Protection Act 1999 (herein referred to as CPA), the United States Uniform Commercial Code 1952, the United States Food, Drugs and Cosmetic Act 1976 and law cases (Ghapheryc \& White, 2012). Whereas the secondary data consisted of journal articles and reports were obtained by browsing law databases such as the Malayan Law Journal, the Current Law Journal, HeinOnline and other databases namely Springer, ScienceDirect, SAGE, Emerald and others. The doctrinal analysis facilitates in the interpretation and understanding of the relationship between different variables adopted by this research namely the pre-market assessment and its application, the Al governance features as well as the integration of Al in the medical setting.

Data analysis approaches adopted by this research include the comparative method and interpretive method. For comparative analysis, this research leverages this method in producing suggestions to improve the current legal position on Al as a medical device (Nelken, 2016). In general, comparative analysis observes a systematic perusal of rules, procedures, institutions, and implementations within single or multiple legal systems based on objective comparative assessments of similarities, differences, and repercussions (Van Hoecke, 2016). A jurisdiction is selected as the country comparison for it provides more legal certainty and a better response to a particular event (Von Mehren et al. 1988). For this research, the United States is selected as the country comparison given the formulation of the research question which emphasises the adequacy of the Malaysian medical device regulation in governing the risk that Al posed to the legal system. In this setting, the researchers' prior knowledge of the United States as the first nation to revamp its pre-market approval system for Al as medical devices is used as the taxonomy of comparison (Pieters, 2009). Interpretive analysis, on the other hand, associates itself with the significance of doctrinal research in formulating legal doctrines through the analysis of legal rules. Unlike scientific research of which the validity of the research findings relies heavily on empirical investigation, the process of analysis by which doctrines are derived gravitates towards more idiosyncratic and argument-based approaches (Chynoweth, 2006). Due to this, the research confides in the definition of 'medical devices' to investigate the competency of the legal system in governing Al from the lens of the medical device regulation. In doing so, the definition of medical devices stipulated under the Medical Device Act 2012 is taken in its broader sense to incorporate the function of Al within the medical context; to diagnose, prevent, treat, provide information for medical or diagnostic purposes and others.

\subsection{Findings}

Distinct from its predecessor disruptive technologies, Al intertwines with the legal system significantly that it has amassed welldeveloped areas of scholarly work and doctrinal support. The literature is replete with discourses on Al and law ever since Al is immensely developed and embraced, with the volume of discussion descents on the question of conventional liability framework and its interwoven effect with the proprietary nature of Al-black box, autonomous trait, self-learning and reinforced learning (Schirmer, 2019) (Gonçalves, 2016) (Balkin, 2015). The chorus of discussion has recently transpired into the deliberation of the institutional competencies in formulating Al policy and governance (Scherer, 2015). Although enacting general laws on Al is unfeasible, it is timely that lights are thrown upon the investigation of Al's current and likely social impacts, and perhaps smaller changes to appropriate doctrines and laws in response to Al's positive and negative affordances (Calo, 2017). Pertinent to this research is the unfolding concept of 'adaptive' Al software as medical devices (SaMD) - where the algorithm learns and improves by itself based on new inputs it acquires during realworld use and experience (U. S. Food and Drug Administration, 2019). Self-adaptative software is pivotal in addressing the dynamic and robustness of human environment, the complexities of managing unexpected conditions, the heterogeneity level of software components as well as the manifold changes of software context, goals and constraints during operation time (Salehie \& Tahvildari, 2009) (Cheng et al., 2014). Correspondingly for AI SaMD, the gleaning of more data to train the system enriches its accuracy and performance within the already convoluted medical landscape. Thus, continual improvement becomes an integral part of the attraction of Machine Learning in creating components of Al systems that read sensor data, text streams, and then adapt the overall behaviour of the Al system accordingly (Bharath, 2019). Nevertheless, since the law is imbued with clarity and precision, the prevalent enthusiasm and dynamism regarding the development of adaptive Al in healthcare are seemingly conflicting. In this setting, scientific understanding and clinical validation are construed as the fundamentals of the medical device regulation in ensuring the safety and efficacy of medical technologies (Pesapane et al., 2018). Unfortunately, adaptive AI SaMD renders these two pillars impracticality. As mentioned before, AI SaMD departs from the typical SaMD with its ability to continuously learn, where the adaptation or change to the algorithm materialises after the system is distributed for use and has acquired real-world experience (Dwivedi et al., 2019).

Following the market distribution, the output derived from this learning and adaptive Al algorithm may deviate from the output initially approved for a predetermined set of application (Kohli et al., 2019) and thus, potentially more risks and less certainty over their benefits. Variance is contributed by software's susceptibility to the interaction with their environment and organisational aspects such as resources, staffing, skills, training, culture, workflow and processes (Gerke et al., 2020). This is further aggravated with Al proprietary black-box; the ability of the system to imitate or produce better decision-making than its human counterparts but without being able to communicate its reasons for doing so, consequently interfering with the scientific understanding required for market clearance (Price, 2017). Clinical validation, on the other hand, verifies that clinically meaningful SaMD output for the predefined use, target health care population and condition recognised in the SaMD definition statement is yielded. Clinically meaningful in this sense signifies that SaMD positive impacts are measurable, patient-centric, and particularly relative to the intended use of AI SaMD for targeted individuals and public health (IMDRF SaMD Working Group, 2017). Every clinical application involves a comprehensive clinical validation to be adopted by the professional clinical community for use in patient care, such as diagnostics or treatment decisions and must be endorsed by 
regulatory authorities. Clinical trials require that the accuracy of the developed Al solution is demonstrated against the clinical standard. The arguments for clinical validation for Al are twofold; 1) the ability of AI SaMD to produce highly personalised treatment predictions or suggestions beyond the artificial testing challenges the clinical validation approaches for medical devices and 2) the diversified standards in ensuring the safety and efficacy of AI SaMD. The first argument reflects the increased variance of AI SaMD because of interacting with physicians and patients progressively, thus possibly allowing the system to recommend more personalised medical outcomes (Gerke et al., 2020). On the other hand, the second argument calls for the accuracy and safety of AI SaMD to be demonstrated against the established clinical standard. However, AI SaMD being at the intersection of interdisciplinary fields of information technology (IT), medical and Artificial Intelligence carries particular certification baggage precisely on the requirement that the development of the device was carried out in conformance with abundant quality standards (Higgins, 2020). Malaysia, in this context, recognised a set of standards to ensure a safe and effective passage of medical devices. Development of or verification for mandatory standards specifically for Al is however absent. It is also uncertain whether the excellent performance of an algorithm is satisfactory if the solution is a "black box" and not transparent and reasonably explainable. In embracing the novel risks posed by Al, it can be presumed that the Malaysian medical device regulation undergone a stagnant development as to the applicability of its pre-market and post-market requirements. There is scarce literature to support the initiatives taken by the Medical Device Authority in revisiting the existing regulatory pathways, probably due to the infant stage of Al adoption in the Malaysian healthcare sector. The United States medical device regulation on the other hand, admittedly, has sustained an extensive development and review of its market approval processes to accommodate emerging technologies in the healthcare sector. Traditionally, the pre-market review pathway outlined by the FDA comprises of the pre-market clearance (510(k), De Novo classification or the Pre-Market Approval depending on the risk that a device demonstrates. However, the FDA presumed that the conventional paradigm of regulating medical devices was not formulated for adaptive Al. That said, under the operating approach to software modifications, a rerun of the pre-market review is called for as many of these Al software are making changes to a device. In 2019, the FDA introduced Regulatory Framework for Modifications to Artificial Intelligence/Machine Learning (Al/ML)-Based Software as a Medical Device, describing FDA's redesigned approaches for Al-related pre-market review, addressing the risk of modifications produced by the technology. The models termed in the regulatory proposal empower practices from FDA's existing pre-market programs and grounded on IMDRF's risk categorization principles, the benefit-risk framework, risk management principles outlined in the software modifications guidance and ultimately, the FDA's organization-based total product lifecycle approach. In light of this framework, FDA envisages a "Predetermined Change Control Plan" embedded in every pre-market submission. This plan delineates the types of anticipated modifications (referred to as the "Software as a Medical Device Pre-Specifications") and the related methodology being employed to implement those changes in a controlled manner that eventually mitigates risks to patients -referred to as the "Algorithm Change Protocol."

\subsection{Discussion}

The conformity assessment under the purview of the Malaysian Medical Device Act 2012 is bound by a two-tier evaluation process by the $C A B$ and the MDA. The MDA acting conjointly with the $C A B$ is mandated with vast power relating to the design of requirements, varying, or revoking conditions of medical device registration. In this respect, the power given mirrors the United States Food and Drugs Administration (FDA)'s broad jurisdiction to modify medical device regulatory controls, impelling the market clearance procedures to be inconsistent and unpredictable. Adversely, the deployment of innovative medical technologies is delayed (Nurus Sakinatul Fikriah et al., 2015). As such, the certainty of market approval and deployment of AI SaMD ought to hail from the deep perception and understanding of the mechanics of medical device regulation, Al and Machine Learning model methodologies and statistical risk evaluation. Higgins (2020) formulated the AI SaMD Regulatory Top-Down Template (as shown in Table 1 below) to determine the audit matrix of AI SaMD. In his proposed guidelines, Higgins acknowledged that while AI SaMD is presumed to demonstrate an initial base performance, modifying its performance parameters based on the input it accumulates is crucial. In this case, regulatory controls should reflect the bounds for the system learning process and strengthened monitoring procedures. This presupposition paralleled the FDA recently introduced Regulatory Framework for Modifications to Artificial Intelligence/Machine Learning (Al/ML)-Based Software as a Medical Device, depicting the FDA groundwork for a potential approach to pre-market review for Al-based SaMD discussed before. The initiative introduced the Total Product Life Cycle regulatory approach by incorporating the FDA benefit-risk framework and risk management principles outlined in the software modifications guidance explicitly addressing the adaptive nature of Al (Minssen et al., 2020).

Table 1: Al SaMD Regulatory Top-Down Template (Higgins, 2020)

\begin{tabular}{|c|c|c|}
\hline Macro Task & Sub-Task & Details \\
\hline Data curation & $\begin{array}{l}\text { Data acquisition } \\
\text { Data storage } \\
\text { Data labelling }\end{array}$ & \\
\hline Adaptation and Modification & $\begin{array}{l}\text { Safe bounds on learning } \\
\text { performance } \\
\text { Appropriate performance /input } \\
\text { monitoring modules }\end{array}$ & $\begin{array}{l}\text { Minimal quality must be } \\
\text { guaranteed. } \\
\text { Existence of a non-adaptiveperformance } \\
\text { monitor. } \\
\text { Robustness analysis of the } \\
\text { non-adaptive performance } \\
\text { monitor. }\end{array}$ \\
\hline In-sample performance & Basic performance metrics & Performance metrics on \\
\hline
\end{tabular}




\begin{tabular}{|c|c|c|}
\hline & $\begin{array}{l}\text { Algorithm properties } \\
\text { perspective } \\
\text { Data properties perspective }\end{array}$ & $\begin{array}{l}\text { hold-out set. } \\
\text { Multi-fold cross-validation } \\
\text { performance. } \\
\text { Calibration (e.g. comparison } \\
\text { with clinical trial data). } \\
\text { Sufficiency of the data set. } \\
\text { Sufficiency of the data at the } \\
\text { clinically relevant changeover } \\
\text { points. } \\
\text { Hyperparameter tuning } \\
\text { procedures. } \\
\text { Expected performance on } \\
\text { 'similar' data (in-distribution). } \\
\text { Handling of input } \\
\text { representations. } \\
\text { Discontinuities. } \\
\text { Missing data, data imputation. } \\
\text { Is convergence assumption } \\
\text { realistic for non-Gaussian } \\
\text { data? } \\
\text { Safe failure modes. }\end{array}$ \\
\hline Post-Market planning & $\begin{array}{l}\text { Shifting clinical standards / } \\
\text { Non-stationarity of data } \\
\text { inputs over time } \\
\text { Surveillance } \\
\text { Software updates }\end{array}$ & $\begin{array}{l}\text { Semantic drift. } \\
\text { New diagnoses. } \\
\text { New input fields. } \\
\text { Telemetry streaming } \\
\text { Paper-based methods. } \\
\text { Certification. } \\
\text { Deployment. } \\
\text { Security/threat model }\end{array}$ \\
\hline
\end{tabular}

\subsection{Conclusion and Recommendations}

Adaptive AI SaMD transforms the way regulators understand the evaluation of safety and efficacy of medical technologies, from which regulatory pathways are formulated. The concept of adaptive AI SaMD against the scientific validation-oriented regulation is brought to light, built upon the premise that the traditional medical device regulation is not designed to address the dynamics of adaptive AI SaMD. This research has highlighted the inadequacy of the pre-market requirements in the Malaysian medical device regulation to govern Al. The premise is grounded on the inability of the existing regulation to appropriately classify Al systems based on its adaptive risks, causing incompatible conformity assessments to be applied in assuring its safety and effectiveness. While the medical device regulation reflects best practices currently employed to facilitate the regulation of medical software, the differences and gaps pertaining Al systems will have to be addressed. In this context, the key to a refined regulation for Al is to produce real world clinical evidence for Al throughout its life cycle, and the possibility for additional clinical evidence to support adaptive systems. From the findings of this research, it is indicated that an all-inclusive conformity assessment for AI SaMD must be a translation of effective communication between medical device regulators and Al experts. Therefore, the design of a regulatory framework should manifest considerations to Al model development and methodologies without discounting the rudimentary quality management system. This research contributes to shed light on the compatibility of the conformity assessment in the Malaysian pre-market requirements for the adaptive nature of $\mathrm{Al}$ and serves as a cornerstone to redesign the potential pre-market approach for adaptive Al in Malaysia. It is hoped that future research will shed light on the triangulation between the doctrinal analysis as well as the contribution from empirical work, precisely on securing Al providers feedback as to the development of a comprehensive regulatory matrix for Al. Additionally, more investigations can be conducted to address other audit matrixes of AI SaMD such as data curation, in-sample performance, post-market planning. This, in turn, facilitates massive development and integration of cutting-edge medical technologies in the Malaysian healthcare landscape through a defined regulatory environment.

\section{Acknowledgement}

The authors would like to express their gratitude for the financial support from Universiti Teknologi MARA under the Lex Praesta Research Grant (600-TNCPI 5/3/DDF (FUU) (001/2020) granted for this research.

\section{Paper Contribution to Related Field of Study}

This research contributes to the chorus of legal interdisciplinary research involving Artificial Intelligence and regulatory science of medical devices. 


\section{References}

Balkin, J. M. (2015). The Path of Robotics Law. California Law Review. 45-60

Benjamens, S., Dhunnoo, P., \& Meskó, B. (2020). The state of artificial intelligence-based FDA-approved medical devices and algorithms: an online database. NPJ Digital Medicine, 3(1), 1-8. https://doi.org/10.1038/s41746-020-00324-0

Bharath, A. (2019). Recent advancements in Al - implications for medical device technology and certification. 1-21. https://www.bsigroup.com/en-GB/medicaldevices/resources/whitepapers/downloads/

Calo, R. (2017). Artificial Intelligence Policy: A Primer and Roadmap. UCDL Review. 399-434

Cheng, B. H. C., De Lemos, R., Giese, H., Inverardi, P., Magee, J., Andersson, J., Becker, B., Bencomo, N., Brun, Y., Cukic, B., Di Marzo Serugendo, G., Dustdar, S., Finkelstein, A., Gacek, C., Geihs, K., Grassi, V., Karsai, G., Kienle, H. M., Kramer, J., ... Whittle, J. (2014). Software engineering for self-adaptive systems: A research roadmap. Lecture Notes in Computer Science (Including Subseries Lecture Notes in Artificial Intelligence and Lecture Notes in Bioinformatics). 1-26 https://doi.org/10.1007/978-3-642-02161-9_1

Chynoweth, P. (2006). Legal Research. 63-71.

Davenport, T., \& Kalakota, R. (2019). DIGITAL TECHNOLOGY The potential for artificial intelligence in healthcare. In Future Healthcare Journal (Vol. 6, Issue 2). 94-98.

Dwivedi, Y. K., Hughes, L., Ismagilova, E., Aarts, G., Coombs, C., Crick, T., Duan, Y., Dwivedi, R., Edwards, J., Eirug, A., Galanos, V.,

llavarasan, P. V., Janssen, M., Jones, P., Kar, A. K., Kizgin, H., Kronemann, B., Lal, B., Lucini, B., ... Williams, M. D. (2019). Artificial Intelligence (Al): Multidisciplinary perspectives on emerging challenges, opportunities, and agenda for research, practice and policy. International Journal of Information Management, July, $0-1$. https://doi.org/10.1016/j.ijinfomgt.2019.08.002

Gerke, S., Babic, B., Evgeniou, T., \& Cohen, I. G. (2020). The need for a system view to regulate artificial intelligence/machine learning-based software as medical device. Npj Digital Medicine, 3(1), 1-4. https://doi.org/10.1038/s41746-020-0262-2

Ghapheryc, J., \& White, E. (2012). Library use of web-based research guides. In Information Technology and Libraries. 21-31 https://doi.org/10.6017/ital.v31i1.1830

Gonçalves, M. A. (2016). Liability Arising from the Use of Artificial Intelligence for the Purposes of Medical Diagnosis and Choice of Treatment: Who Should be Held Liable in the Event of Damage to Health? 1-75.

Higgins, D. (2020). On Regulating Al in Medical Products (OnRAMP). ArXiv: Computers and Society. 1-23 http://arxiv.org/abs/2010.07038

Hutchinson, T., \& Duncan, N. (2014). Defining and Describing What We Do: Doctrinal Legal Research. 1(1988).

IMDRF SaMD Working Group. (2017). Software as a Medical Device (SaMD): Clinical Evaluation. International Medical Device Regulators Forum, September, 4-8.

Jarow, J. P., \& Baxley, J. H. (2015). Medical devices: US medical device regulation. In Urologic Oncology: Seminars and Original Investigations https://doi.org/10.1016/j.urolonc.2014.10.004

Jiang F, Jiang Y, Zhi H, et al. (2017). Artificial intelligence in healthcare: past, present and future. Stroke and Vascular Neurology. 230-243 .https://doi.org/10.1136/svn-2017-000101

Kramer, D. B., Xu, S., \& Kesselheim, A. S. (2012). How does medical device regulation perform in the United States and the European union? A systematic review. In PLoS Medicine. https://doi.org/10.1371/journal.pmed.1001276

Lohr, S. (2016). IBM Is Counting on Its Bet on Watson, and Paying Big Money for It - The New York Times.

Medical Device Authority. (2014). Conformity Assessments. 2014. https://portal.mda.gov.my/industry/medical-device- registration/general-medical-device.html\#Regulate

Meskó, B. (2020). OPEN A short guide for medical professionals in the era of arti fi cial intelligence. Npj Digital Medicine. 1-8. https://doi.org/10.1038/s41746-020-00333Minssen, T., Gerke, S., Aboy, M., Price, N., \& Cohen, G. (2020). Regulatory responses to medical machine learning. Journal of Law and the Biosciences. 1-18. https://doi.org/10.1093/jlb/lsaa002

Naudé Fourie, A. (2015). Expounding the Place of Legal Doctrinal Methods in Legal-Interdisciplinary Research. Erasmus Law Review. https://doi.org/10.5553/elr.000045 Nelken, D. (2016). Comparative Legal Research and Legal Culture: Facts, Approaches, and Values. In Annual Review of Law and Social Science. https://doi.org/10.1146/annurev-lawsocsci-110615-084950

Nurus Sakinatul Fikriah, M. S. P., Hartini, S., \& Zulhabri, I. (2015). The Medical Device Regulation for Humanoid Robotics: Does One Size Fits All? Procedia Computer Science, 76(December), 381-387. https://doi.org/10.1016/j.procs.2015.12.313

Otake, T. (2016). IBM big data used for rapid diagnosis of rare leukemia case in Japan | The Japan Times.

Pesapane, F., Volonté, C., Codari, M., \& Sardanelli, F. (2018). Artificial intelligence as a medical device in radiology: ethical and regulatory issues in Europe and the United States. In Insights into Imaging. 745-753 https://doi.org/10.1007/s13244-018-0645-y

Pieters, D. (2009). Functions of comparative law and practical methodology of comparing. Research Master in Law., 1-35. Price, W. N. (2017). Regulating black-box medicine. Michigan Law Review. 420-474. 
Salehie, M., \& Tahvildari, L. (2009). Self-adaptive software: Landscape and research challenges. ACM Transactions on Autonomous and Adaptive Systems, 4(2), 1-40. https://doi.org/10.1145/1516533.1516538

Savage, N. (2020). How Al is improving cancer diagnostics. In Nature (Vol. 579, Issue 7800, pp. S14-S16). NLM (Medline). https://doi.org/10.1038/d41586-020-00847-2

Scherer, M. U. (2015). Regulating Artificial Intelligence Systems: Risks, Challenges, Competencies, and Strategies. SSRN Electronic Journal, 29(2). https://doi.org/10.2139/ssrn.2609777

Schirmer, J. E. (2019). Artificial intelligence and legal personality: Introducing "Teilrechtsfähigkeit": A partial legal status made in

Germany. In Regulating Artificial Intelligence. https://doi.org/10.1007/978-3-030-32361-5_6 Shiffman, J. (2018). The Critical Role of Regulatory Affairs in the Medical Device Industry. 2018. https://www.northeastern.edu/graduate/blog/regulatory-affairs-in-medical-device-industry/

U. S. Food and Drug Administration. (2019). Artificial Intelligence and Machine Learning in Software as a Medical Device | FDA. https://www.fda.gov/medicaldevices/software-medical-device-samd/artificial-intelligence-and-machine-learning-software- medical-device

US Food and Drugs Administration. (2017). Global Approach to Software as a Medical Device. 2017. https://www.fda.gov/medical- devices/software-medical-devicesamd/global-approach-software-medical-device

Van Hoecke, M. (2016). Methodology of Comparative Legal Research. Law and Method, June 2015. https://doi.org/10.5553/rem/.000010

von Mehren, A. T., Weir, T., Zweigert, K., \& Kotz, H. (1988). An Introduction to Comparative Law. The American Journal of Comparative Law. $495-497$. https://doi.org/10.2307/840281

World Health Organisation. (2015). Medical Device Regulation: Global Overview and Guiding Principles (Vol. 5, Issue July 2013). 